\title{
Designing Future Underwater Vehicles: Principles and Mechanisms of the Weakly Electric Fish
}

\author{
Malcolm A. MacIver, Ebraheem Fontaine, and Joel W. Burdick
}

\begin{abstract}
Future underwater vehicles will be increasingly called upon to work in cluttered environments and to interact with their surroundings. These vehicles will need sensors that work efficiently at short range and be highly maneuverable at low speed. To obtain insights into principles and mechanisms of low-speed operation in cluttered environments, we examine a fish that excels in this regime, the black ghost knifefish Apteronotus albifrons. This fish hunts in dark or turbid water using a short-range self-generated electric field to sense its surroundings. Coupled with this unique mode of sensing is an unusual ribbon fin propulsion system that confers high multidirectional maneuverability at low speeds. To better understand the relationship between body morphology and common maneuvers of this fish, we utilized an idealized ellipsoidal body model, Kirchhoff's equations, and an optimal control algorithm for generating trajectories. We present evidence that common fish trajectories are optimal, and that these trajectories complement the sensory abilities of the fish. We also discuss prototypes of the sensing and propulsion systems of the fish with a view to providing alternative approaches for underwater vehicle design where high maneuverability in geometrically complex environments is needed.
\end{abstract}

Index Terms-Active sensing, autonomous underwater vehicles, backward swimming, biologically inspired robotics, biorobotics, electric fish, electrosensory, gymnotiform, knifefish, locomotion, maneuverability, remotely operated vehicles, ribbon fin.

\section{INTRODUCTION}

$\mathbf{T}$ HIS paper examines an unusual fish species, the weakly electric black ghost knifefish (Apteronotus albifrons) (Fig. 1), whose sensory system, propulsion scheme, and body design principles may be relevant for next generation autonomous underwater vehicles (AUVs). Traditionally, underwater robots are grouped into two main categories: AUVs, which operate for relatively long periods without human guidance, and remotely operated vehicles (ROVs) that are powered and teleoperated via a tether connected to a surface command ship. AUVs are typically used for data gathering in the open ocean, and they rarely have physical interactions with their surroundings. ROVs, on the other hand, often work in closer quarters and deploy robot arms or other tools that interact

Manuscript received July 18, 2003. This work was supported by the Engineering Research Centers Program, National Science Foundation, under Award EEC-9402726.

M. A. MacIver was with the Division of Engineering and Applied Science and the Computation and Neural Systems Program, California Institute of Technology, Pasadena, CA 91125 USA. He is now with the Departments of Mechanical and Biomedical Engineering, Northwestern University, Evanston, IL 60208-3111 USA (e-mail: maciver@northwestern.edu).

E. Fontaine and J. W. Burdick are with the Division of Engineering and Applied Science, California Institute of Technology, Pasadena, CA 91125 USA (e-mail: ebraheem@robotics.caltech.edu; jwb@ robotics.caltech.edu).

Digital Object Identifier 10.1109/JOE.2004.833210

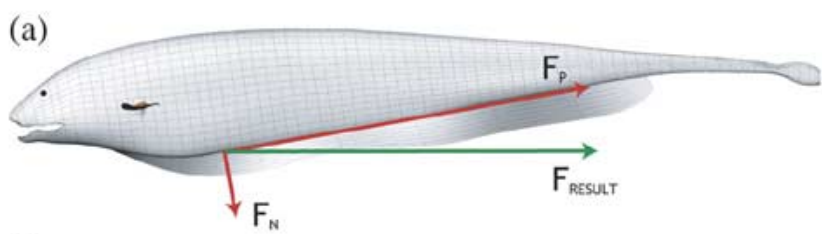

(b)
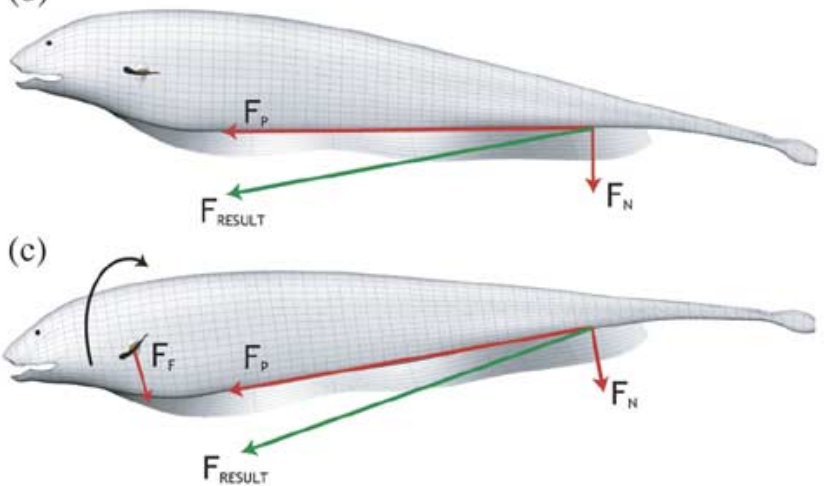

Fig. 1. Hypothesized (see [11]) thrust vectors around Apteronotus albifrons for two swimming directions and a roll maneuver. $\mathrm{F}_{N}$ is force normal to line of ventral ribbon fin; $\mathrm{F}_{P}$ is the force parallel to this fin, and $\mathrm{F}_{F}$ is the force generated by pectoral fin. (a) Swimming forward. Pectoral fins are typically held at the indicated angle of attack during this behavior. (b) Swimming backward. Note that the body needs to be negatively pitched to go straight back. We have observed such negative pitching of the body during straight-back swimming (unpublished observations). (c) Swimming up and back with a roll by forcing fluid down on one side of the body, similar to the motion observed during a stereotypical prey strike (see Fig. 2).

closely with nearby objects. However, they currently exhibit very low levels of autonomy and mediocre hydrodynamic efficiency. Future underwater vehicles will be increasingly called upon to work in cluttered environments and interact with their surroundings like ROVs, while exhibiting the autonomy and efficiency of AUVs. Examples of future tasks include autonomous exploration of the interiors of sunken vessels, or autonomous maintenance operations on underwater oil wells. Biological propulsion and sensing paradigms continue to have a competitive edge over current underwater vehicle technology in the domain of low-speed, highly maneuverable, and efficient operation in cluttered environments. Therefore, the study of fish species that are specially adapted to such environments, in terms of both their propulsion and sensing abilities, may provide inspiration for better AUV technology as well as clues about how to integrate sensing and mechanics in the design of vehicles for complex environments.

In Section II, we consider some of the challenges of current underwater vehicle design to highlight future design needs. In Sections III-VI, we introduce weakly electric fish, describe stereotypic maneuvers that have been quantified in previous 


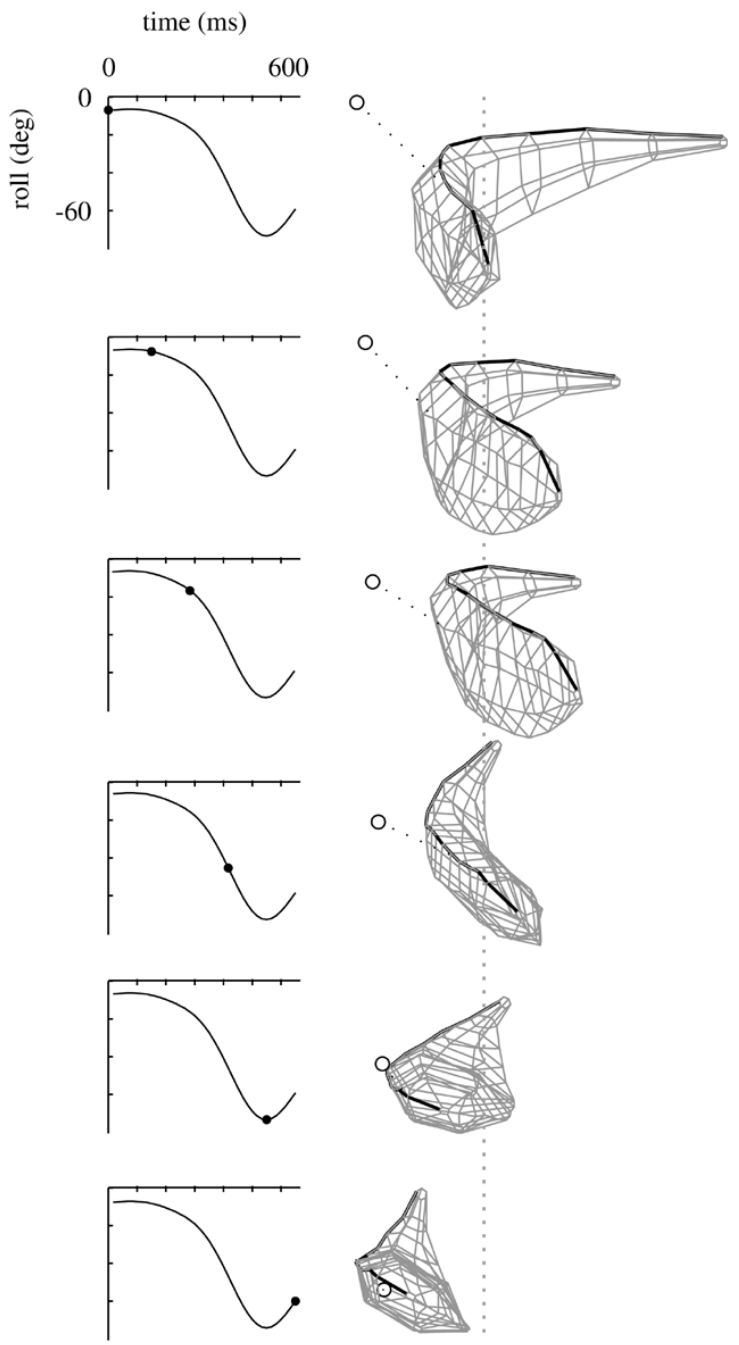

Fig. 2. A typical rolling maneuver to reach a laterally positioned prey. Wireframe represents position of fish as it strikes a prey, from 3-D motion capture data. The top snapshot $(t=0 \mathrm{~ms})$ is at the time of prey detection, and time increases up to the last snapshot at the end of the sequence. The heavy line on the top edge of the fish indicates the dorsal edge, the open circle marks the position of the Daphnia magna, and the dotted line indicates the shortest distance from the Daphnia to the body surface. The inset plot on the left shows the roll angle history and current value (filled circle). (Modified from [5].)

work on this fish, and examine these maneuvers through the methods of optimal control theory. In summary, we show that the animal's behavioral strategies for prey capture are highly influenced by considerations of fluid mechanical efficiency. We wish to stress two messages here. First, although robots are intended to be multipurpose mechanisms, they are most often deployed for a limited number of stereotypical tasks. Thus, in selecting their design, it obviously makes senses to optimize for the robot's most frequently encountered operations. The second message is that for short-range motions, which will be typical of autonomous vehicle operations in complex environments, simple potential flow models coupled with optimal control principles can provide a computationally tractable set of tools for underwater vehicle design optimization. In Section VII, we interpret the optimal control results with consideration of what is known about the sensing and movement abilities of these fish. We demonstrate that the sensory and locomotor capabilities are closely integrated to execute the stereotypic maneuvers.
Although such integration should always be the aim of good system design, the detailing of this integration in a biological system is both inspiring and informative. In Sections VIII and IX, we describe some initial work on developing a robotic electrosensory array and robotic ribbon fin for use on underwater vehicles. We conclude with some comments on the broader AUV design implications of this research.

\section{Challenges of Current Underwater VEHICLE TECHNOLOGY}

Many AUVs (e.g., WHOI's REMUS and MIT's Odyssey) have used the same basic design principle. A propeller thruster is combined with control fins to propel and steer the vehicle. Maneuvering control forces are generated by fluid flow over control surfaces, and the torpedo-shaped body is optimized for low drag during forward motion. These vehicles also use powerful sonars to sense their surroundings. At cruising speeds, and for relatively uncluttered spaces, this combination of mechanical design and long-range sensing is extremely efficient and effective. However, because these vehicles are controlled by forces that are based on lift, they are difficult to maneuver at low speeds or in tight quarters. Moreover, their sonar sensors are not particularly effective at very close range, and therefore they would fail to provide useful navigation data in cluttered close quarter situations, such as the exploration of a sunken vessel interior.

ROVs (such as WHOI's JASON [1] and MBARI's Tiburon) achieve better low-speed hovering and maneuverability abilities through a "box design" or a multipontoon design, coupled with the use of multiple thrusters. Some AUVs (such as Stanford's OTTER [2] and WHOI's ABE and SeaBED) also adopt these body plans. Although the multithruster bulky body design leads to superior hovering and maneuvering abilities relative to torpedo-like AUVs, even these vehicles have problems with station keeping or precision maneuvering in low speed drifting currents. Propellers are excellent when they operate at constant speed. However, for small motion corrections, current ROV control practice leads to "twitching" motions of propellers that involve less than a full shaft rotation. In this unsteady fluid regime, propellers are less efficient, and the resulting fluid impulse is more difficult to predict. This results in degraded control precision and possibly periodic oscillations of the vehicle's position. Moreover, whereas the high drag of the bulky body design naturally improves hovering ability, it reduces hydrodynamic efficiency. More importantly, ROVs currently exhibit little autonomy, and therefore there has been little development of sophisticated sensors that can map the vehicle's immediate surroundings for purposes of autonomous obstacle avoidance and vehicle navigation. At present, television cameras, which require energy-intensive lighting [3], are the primary navigation sensors.

In order to achieve an AUV with ROV capabilities, a propulsion method that provides thrust at low velocities is needed, as well as a sensing system that works efficiently in cluttered environments. For high efficiency, these sensing and actuation technologies need to be well integrated with the typical maneuvers the vehicle needs to make. In the following, we show how these considerations are satisfied in weakly electric fish. 


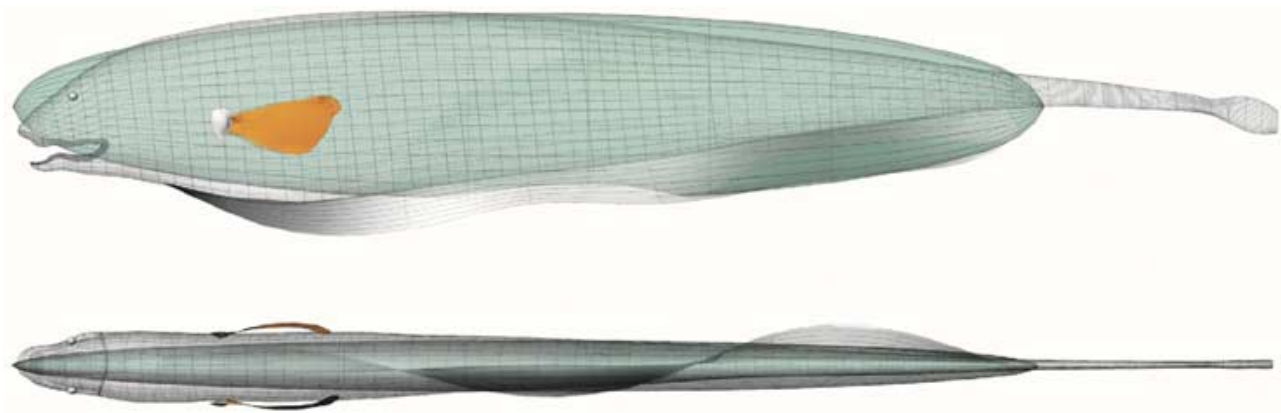

Fig. 3. Body plan of the black ghost Apteronotus albifrons overlaid with the ellipsoidal model.

\section{WEAKLY ELECTRIC FISH}

The black ghost knifefish is a highly active, predatory fish that hunts in the rivers of the Amazon basin at night and hides during the day. Because it hunts at night, and in turbid waters, it has evolved a specialized system to sense its immediate surroundings via a weak $\left[1 \mathrm{mV} \mathrm{cm}^{-1}\right.$ near the body, self-generated electric field. Nearby objects that differ in electrical conductivity from the surrounding water create localized voltage perturbations that are sensed by $\approx 14000$ [4] voltage-sensitive sensory organs scattered over the body surface. The range of electrosense is about one third of the body length for small targets such as water insects, and about one body length for larger objects [5]. Adult fish are $15-50 \mathrm{~cm}$ in length. In addition to their specialized sensory capabilities, gymnotiform knifefish possess a unique multidirectional propulsion system driven by a ventral ribbon fin that runs most of the length of the body (Fig. 1). By generating traveling waves along the ribbon fin and manipulating the pectoral fins, they can move forward, backward, and upward; they can also rapidly pitch or roll the body [5]-[9]. Although the mechanism of thrust production for this fin is not yet known, it is clear that the fin provides precision positioning at low velocities. For example, the fish typically detects 3-mm-long prey in the tail region while swimming forward at $10 \mathrm{~cm}-\mathrm{s}^{-1}$, and it is able to reverse and capture this small prey in less than one second [5]. Because the sensory system of weakly electric fish has been the subject of decades of investigation [10], they provide unique opportunities for examining the codesign of sensing and actuation.

\section{WeAKLy EleCtric Fish MaNeUvers}

During prior behavioral studies [5], individual black ghost knifefish were videotaped hunting for small prey under infrared light within a light-tight enclosure. Two cameras provided orthogonal views of the behavior. Prey-capture sequence video was digitized and brought into a custom model-based tracking system [13]. A nonrigid wireframe model of the fish was overlaid onto the video in the two views, and a three-dimensional (3-D) reconstruction algorithm provided the position of the surface of the fish and prey to $\pm 0.5 \mathrm{~mm}$, with a time resolution of $16.7 \mathrm{~ms}$ [5], [13]. A single Daphnia magna, a small (up to $3 \mathrm{~mm}$ in length) crustacean that these fish are known to eat in their native habitat, was utilized for each trial.

1) Rapid Reversal Maneuver: Prey were typically detected while the fish swam forward [Fig. 1(a)]. Following the detection, the fish began to decelerate. We used the onset of deceleration as an estimate of the time of prey detection. Deceleration was very rapid (from around $10 \mathrm{~cm}-\mathrm{s}^{-1}$ to $-20 \mathrm{~cm}-\mathrm{s}^{-1}$ over the course of $300 \mathrm{~ms}$ [5]). It was mediated by a combination of reversing the direction of the traveling waves on the ribbon fin [Fig. 1(b)] and one or two synchronous high-speed backward rowing motions of the pectoral fins (which have the characteristic paddle shape seen in highly maneuverable fish; see [14]). We refer to this as a "rapid reversal" maneuver.

2) Dorsal Roll Maneuver: Following prey detection, if the prey was to the side of the body, the body would roll (around its long axis) approximately the angle to the prey so that the prey would be centered directly above the dorsal (top) edge of the body [5] (Fig. 2). Simultaneously, it would reverse and slice through the water with the narrow dorsal edge of the body leading. The body was maintained in a rigid, straight conformation during this behavior, with roll, pitching, and translation occurring through a combination of ventral ribbon fin activity and manipulation of the pectoral fins. For example, by moving backward with ribbon fin motion, in combination with holding the left pectoral fin at an angle of attack so as to create an upward pressure on the left fin (by pushing fluid downward as it moves by), the body will roll to the animal's right [Fig. 1(c)]. Such a force-torque pair, also created to change the pitch angle of the fish's body (but with bilaterally symmetric upward or downward pressure through the two pectorals), is referred to as a "wrench" in mechanics. We refer to this as a "dorsal roll" maneuver. Typically, detected prey were positioned above the animal [5]; thus, the upward thrust gained from reversing the fin [see force vectors in Fig. 1(c)] is advantageous to the animal.

\section{FluID AND BODY MODELS}

To analyze the relationship between the fish's body plan, its fluid environment, and its stereotypical behavior, we constructed a simple fluid model that captured the key dynamical properties that are relevant for prey-capture maneuvers. We combine this model with an optimal control analysis in the next section in order to assess how much of the observed behavior and sensor geometry can be attributed to purely fluid mechanical effects.

We idealized the knife-like fish body as a rigid ellipsoid of similar length $(13 \mathrm{~cm})$, depth $(2.4 \mathrm{~cm})$, width $(0.64 \mathrm{~cm})$, surface area $\left(52.1 \mathrm{~cm}^{2}\right)$, volume $\left(10.1 \mathrm{~cm}^{3}\right)$, and mass $(10.1 \mathrm{~g})$ as the fish used in prior behavioral studies [5]. Fig. 3 shows the black ghost knifefish body plan alongside the approximating ellipsoid. For this analysis, we consider the ellipsoid to be submerged in an 
ideal fluid (inviscid, incompressible, irrotational, and at rest at infinity). For the Reynolds number typical of this fish $\left(10^{3}-10^{4}\right.$ [9]), the inviscid assumption is reasonable. In addition, for the short-range, transient maneuvers considered here, the acceleration reaction forces we model should dominate, and correlate with, the effects of drag. These "added mass" effects are forces due to the fluid that is accelerated and displaced by movement.

Finally, the behaviors of the fish we will be investigating with this model (body roll and rapid reversals of the direction of movement) are often executed with a rigid straightened body, or if the body is bent before the maneuver, the bend rapidly decreases during the maneuver [5]. Therefore, the absence of bending in our ellipsoidal approximation is appropriate for modeling these behaviors. Rigidity of the body of the knifefish during locomotion has long been noted and is in part mediated by a special body-stiffening adaptation, the presence of intramuscular bones [15], [16].

Inasmuch as the fish is able to maneuver without bending the body by use of the ribbon fin and pectoral fins (neither of which possess sensors), it is able to decouple locomotory movements from active sensing body movements [5]. This in turn may reduce the reliance on "reafference suppression"-removal of self-induced stimulation due to movements of the electric organ inside of the body. Such movements can cause much larger modulations of sensory nerve activity than those caused by prey [5], [17].

The equations of motion for an ellipsoid in ideal fluid are Kirchhoff's equations [18], [19]

$$
\begin{aligned}
\dot{\boldsymbol{\omega}} & =\boldsymbol{J}^{-1}(\boldsymbol{J} \boldsymbol{\omega} \times \boldsymbol{\omega}+\boldsymbol{J} \boldsymbol{v} \times \boldsymbol{v}+\mathbb{T}) \\
\dot{\boldsymbol{v}} & =\boldsymbol{M}^{-1}(\boldsymbol{M v} \times \boldsymbol{\omega}+\mathbb{F})
\end{aligned}
$$

where $\boldsymbol{\omega}$ and $\boldsymbol{v}$ are vectors of the angular and linear velocities of the ellipsoid, respectively, in a body-fixed coordinate frame, $\boldsymbol{J}$ is the sum of the inertia matrix of the body and the added inertia matrix due to the effect of the fluid displaced by rotations, $M$ is the sum of the mass matrix of the body and the added mass matrix due to the fluid displaced by translations, and $\mathbb{T}$ and $\mathbb{F}$ are vectors of the external torques and forces, respectively. The axes of the body-fixed frame are chosen to coincide with the principal axes of the ellipsoid; thus the mass and inertia matrices are diagonal. The elements $m_{i}$ of the diagonal mass matrix $\boldsymbol{M}$ are computed as [18], [19]

$$
m_{i}=\rho V\left(1+\frac{\gamma_{i}}{2-\gamma_{i}}\right)
$$

where

$$
\begin{aligned}
\gamma_{i} & =l_{1} l_{2} l_{3} \int_{0}^{\infty} \frac{d \lambda}{\left(l_{i}^{2}+\lambda\right) \Delta} \\
\Delta & =\sqrt{\left(l_{1}^{2}+\lambda\right)\left(l_{2}^{2}+\lambda\right)\left(l_{3}^{2}+\lambda\right)}
\end{aligned}
$$

where $l_{i}$ is the length of the semiaxes of the ellipsoid along the $i$ th principal axis for $i=1,2,3(6.48,1.18$, and $0.32 \mathrm{~cm}$, respectively), the volume of the ellipsoid is $V=4 \pi l_{1} l_{2} l_{3} / 3$, and $\rho$ is the density of water $\left(1000 \mathrm{~kg}-\mathrm{m}^{-3}\right)$. This gives $\left(m_{1}, m_{2}, m_{3}\right)=$ $(10.4,12.9,45.9) \mathrm{g}$ (effective mass moving forward, up, and lat- erally, respectively). The element $j_{1}$ of the diagonal inertia matrix is

$$
j_{1}=\frac{1}{5} \rho V\left(l_{2}^{2}+l_{3}^{2}+\frac{\left(l_{2}^{2}-l_{3}^{2}\right)^{2}\left(\gamma_{3}-\gamma_{2}\right)}{2\left(l_{2}^{2}-l_{3}^{2}\right)+\left(l_{2}^{2}+l_{3}^{2}\right)\left(\gamma_{2}-\gamma_{3}\right)}\right)
$$

with $j_{2}$ and $j_{3}$ cyclic permutations of the formula for $j_{1}(1 \mapsto$ $2 \mapsto 3)$. This gives $\left(j_{1}, j_{2}, j_{3}\right)=(7.5,362.2,108.6) \mathrm{g} \mathrm{cm}$ (effective inertia rotating around the long axis, around the vertical axis, and around the transverse axis, respectively).

\section{OPTIMAL CONTROL}

\section{A. Dorsal Rolls and Rapid Reversals}

One way to approach animal behavior is to devise a quantitative measure of the "effort" of movement and determine whether the behavior minimizes this effort. This is the basis for applying optimal control theory in the context of the previously described fish behaviors. Dorsal rolls and rapid reversals are a nearly ubiquitous feature of the prey-capture sequences we quantified in $A$. albifrons [5], and they are therefore good behaviors to examine for optimality.

Using the ellipsoidal model of the fish in an ideal fluid, we examined whether mechanical factors alone may determine the rolling and rapid reversal maneuvers. That is, without regard to sensing, control issues, or actuation (i.e., the ellipsoid is fully actuated, unlike the fish), would the ellipsoid exhibit qualitatively similar behavior to what we observed in the real fish? Further, can the optimal control analysis explain the sensor distribution on the body?

\section{B. Synthetic Trajectory Generation}

Optimal trajectories for the ellipsoidal body were generated using an optimal trajectory generation algorithm [20], with custom software developed by Milam [21]. The algorithm finds a trajectory that satisfies (1) and (2) while minimizing a given cost functional subject to a set of trajectory constraints. The constraints specify initial and final conditions of the system's state variables and controls, as well as trajectory constraints such as that a certain level of force not be exceeded or that the pitch of the body not exceed some maximum value. In our case, we chose a "control cost" functional

$$
J=\int_{t_{0}}^{t_{f}}\|\boldsymbol{u}(t)\|^{2} d t
$$

where $t_{0}$ is the initial time, $t_{f}$ is the final time, $\boldsymbol{u}$ is the $(\mathbb{T}, \mathbb{F})$ of (1) and (2), a set of six torque and force control vectors $\left(t_{x}, t_{y}, t_{z}, f_{x}, f_{y}, f_{z}\right)$. There were no penalties for torques or forces in any of the degrees of freedom. The control cost of a trajectory for the ellipsoid can be thought of as analogous to the metabolic cost of muscle activation that would be required for a fish to execute the trajectory.

For trajectory constraints, initial and final conditions were chosen as described below, subject to two global constraints: Euler angles of the body (yaw, pitch, and roll) were bounded to be $\geq 0$ and $\leq 2 \pi$ over the course of the trajectory, and the trajectory must end with the ellipsoid at zero velocity. We chose this because at the end of prey capture trajectories of A. albifrons, 
the fish's velocity is near zero [5]. We did not constrain the final angular velocity, or positional and angular accelerations.

We utilized two different sets of initial and final conditions. The first was designed to be similar to conditions under which a "dorsal roll" is observed during prey capture, and under which we observe "side-scanning" behavior where the fish will scan the substrate for food, moving dorsal-edge first with the side of the body parallel to the bottom. This initial condition specified a zero initial velocity and a final position about a body length directly lateral of the initial position. The second initial condition was designed to investigate the "rapid reversal" strategy. We set the forward velocity to near the mean of the searching velocity of the fish (prior to prey detection) $\left(10 \mathrm{~cm} \mathrm{~s}^{-1}\right)$ and the pitch of the ellipsoid to the mean pitch during searching behavior $\left(30^{\circ}\right)$ [5], with a final position slightly dorsal and to the side of the initial position. This approximates the configuration of the fish and prey during a near head-on detection.

We found that the optimal trajectories, given these initial conditions, were qualitatively very similar to those quantified for weakly electric fish. Both the rolling behavior and "rapid reversal" aspects of the kinematics [5] were found (Fig. 4). An additional aspect that was also previously documented, pitch reduction during the reversal [5], was also observed in the optimal trajectories [Fig. 4(b), right column]. In the future, we will be performing more detailed, trial-by-trial comparisons of the fish trajectories and the optimal control trajectories. Although other cost functionals can be analyzed, this "metabolic-effort" cost functional is likely to be the most important.

\section{Codesign of Body Plan, Sensor GeOMEtry, CONTROL, AND MECHANICS}

The complementarity of sensing to locomotion is an ancient pattern stretching back to the early appearance of the bilateral animal body plan. In this body plan, bilateral symmetry, which often [22] includes high forward mobility along the midline axis, is closely coupled with a clustering of sensory organs around the head. All vertebrates and most other highly mobile animals such as insects feature this body template. With this template came the active feeding behaviors and agile locomotion that correlate with the evolution of advanced nervous systems needed to control these behaviors [23]-[25].

In the specific case of the weakly electric fish, the optimal control results help interpret the layout of sensors on the body, including higher sensor densities on the dorsal edge as well as in the head region [4]. Thus, by rolling the dorsal edge toward objects, the fish is not only entering a mechanically optimal maneuver, it is also increasing the quantity and resolution of sensory inflow. Similarly, rapidly reversing the body, in addition to being the mechanically optimal approach for objects behind the fish, brings targets into the head region, where there is an order of magnitude higher density of receptors than on the rest of the body.

In addition to its sensing and mechanical advantages, the rolling maneuver may also simplify the control problem the brain of the fish has to solve: by centering the prey between the bilaterally symmetric halves of its electrosensory system, the fish could be executing a simple localization strategy (sometimes referred to as a "tropotaxis"[26]). To execute this strategy, the fish would first roll its body to null any imbalance in input between the two sides of its sensory system; then it would climb the gradient of sensory signal strength until capture occurs [5]. Another control problem the rolling maneuver helps solve is that it allows the fish to move in unactuated directions. Although the fish has no way to translate laterally, it can move in that direction by first rolling $90^{\circ}$ and then swimming through the water with its dorsal edge first, similar to observed motions (Fig. 2) and the optimal maneuver shown in Fig. 4(a).

From our 3-D motion capture data, we have measured remarkably high roll angular velocities in excess of $400^{\circ} \mathrm{s}^{-1}$, indicating that this maneuver can be rapidly engaged. The torque needed by the animal to roll the body will be proportional to its moment of inertia along the highly stable longitudinal axis. As computed above, the ellipsoidal approximation of the body has a very low moment of inertia along the longitudinal axis, an order of magnitude lower than rotations around either of the other two axes of the body. Thus, rolling is facilitated by the long tapered shape of the body. This is in contrast to other fish, where body roll is stabilized by deep, laterally compressed body shapes [27].

These considerations point toward a high level of interdependency between the body plan, sensor geometry, and observed maneuvers in weakly electric fish. We have recently found evidence of a further interdependency between the space that the fish can rapidly move to and the space that objects can be detected within. Both spaces are cylinder-like shapes around the body, providing an omnidirectional sensing envelope that is complemented by an omnidirectional movement envelope. Further, the fish appears to be investing just enough energy into its sensory field to be able to detect small prey and come to a stop before colliding with them. This matching of sensory ability to locomotor ability and behavioral needs is another example of how these systems have been mutually tuned to optimize the power efficiency of the system.

Although it is tempting to consider mechanics as the causal antecedent of the sensor geometry and body plan, in reality the high level of integration of sensing, locomotion, and simple control strategies enabled by bilateral symmetry and body roll likely reflects the coevolution of these different elements. As has been previously pointed out [28]-[32], given the tight coupling occurring in these systems, situating the locus of control in either the nervous system or mechanical system may not be possible, with each playing a part to an extent that varies with the mode of locomotion, velocity, and the degree of sensory challenge posed by the environment, among other factors [29], [31].

\section{Electrosense as a Possible AUV SENSORY MODALITY}

The use of weak electrostatic fields to accomplish short-range sensing has received little attention from underwater vehicle engineers. It offers several advantages, however, including the ability to function in turbid waters and low power consumption. To explore the feasibility of creating an artificial electrosensory system for underwater robots, in earlier work with Nelson [33], 


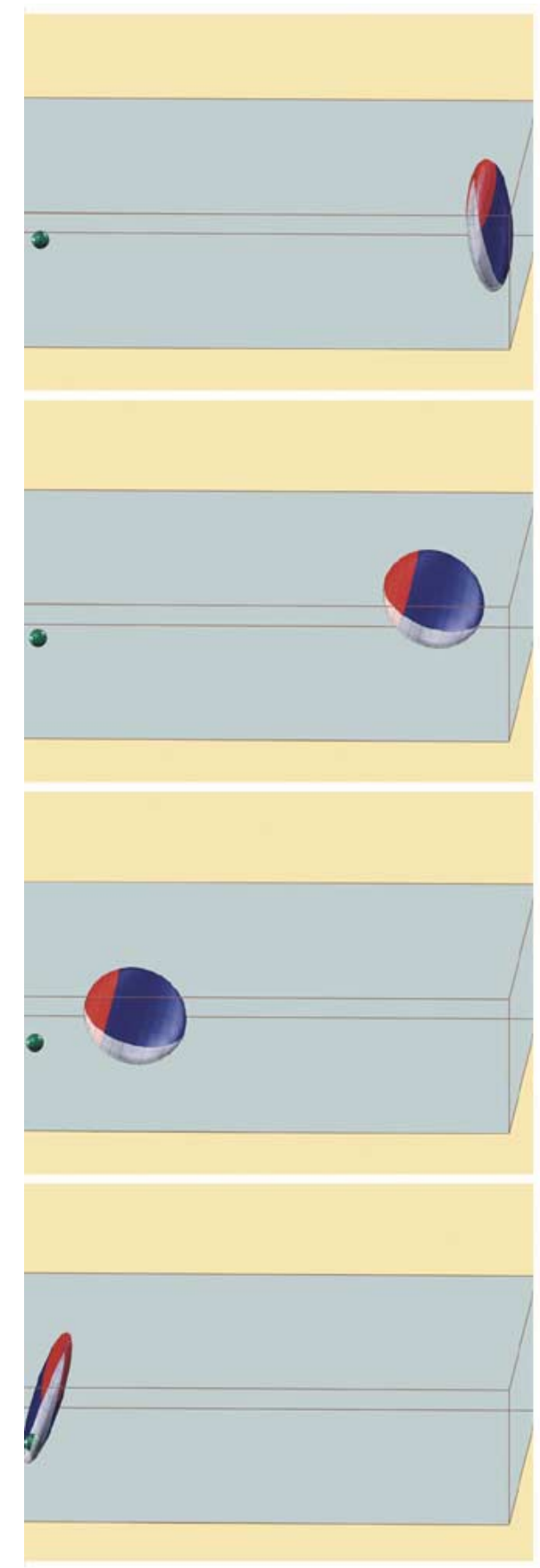

(a)

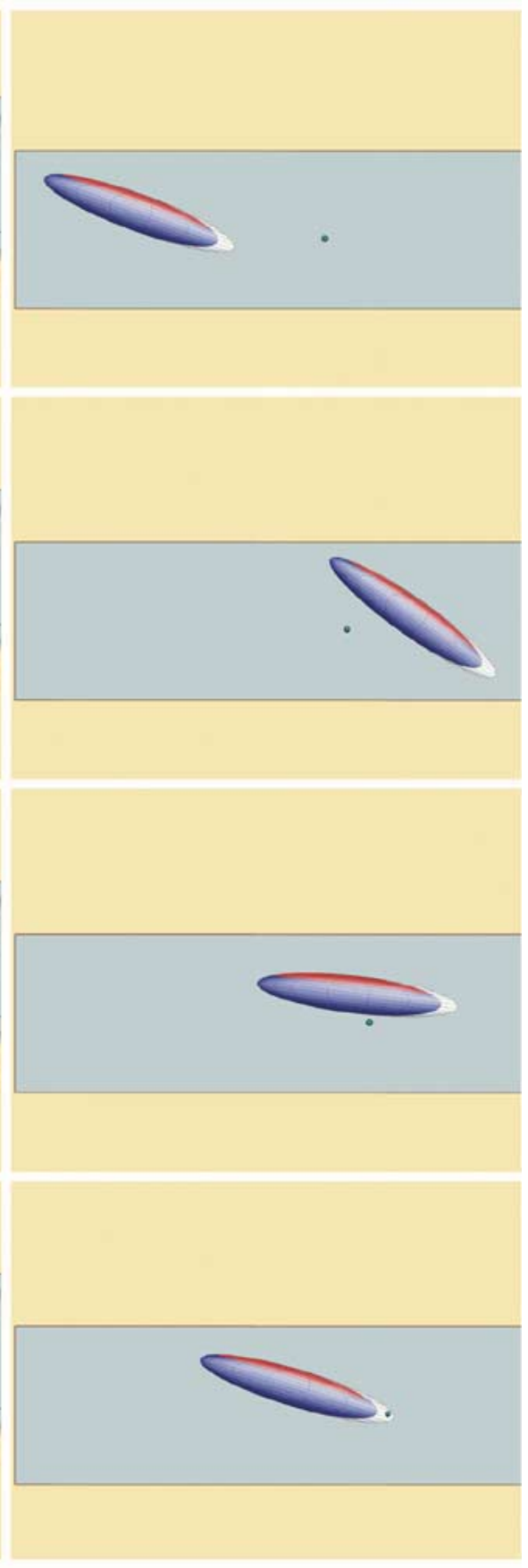

(b)

Fig. 4. Snapshots of control cost optimal trajectories for the two initial conditions representing common electric fish prey-capture maneuvers, with time increasing from top to bottom. Final position indicated by the small sphere ("prey") outside of the ellipsoid. (a) View (from close to head-on) of the ellipsoid rolling into the fluid as it performs a lateral translation. (b) Side view of the ellipsoid moving forward (to the right). As it moves forward, the pitch angle decreases, and the ellipsoid performs a reversal to translate to the prey.

we developed a small linear active electrosensory array and a three-axis robotic workcell for controlling the movement of a target object near the array [33], shown in Fig. 5.

This prototype system allowed us to acquire and analyze electrosensory signals similar to those experienced by weakly electric fish. Studies by Rasnow [34] have shown that the electrosensory image of a small spherical object is spatially broad and weak for distant objects, becoming sharper and stronger as the object approaches the fish. To examine whether our artificial active electrosensory system exhibited similar voltage patterns, a test object (a 1-cm-diameter plastic sphere) was scanned parallel to the sensor array at four different distances from the array (6, 9,12 , and $15 \mathrm{~mm}$ ) with a velocity of $4 \mathrm{~cm}-\mathrm{s}^{-1}$. Distances were measured from the array to the center of the test object. As illustrated in Fig. 5, the signal profiles obtained from the artificial sensor array were qualitatively similar to those observed in electric fish. The voltage signal is strong and narrow when the object is close to the array and becomes weaker and broader as the target distance is increased. Future studies will explore these relationships for the artificial array in more quantitative detail. 
(a)

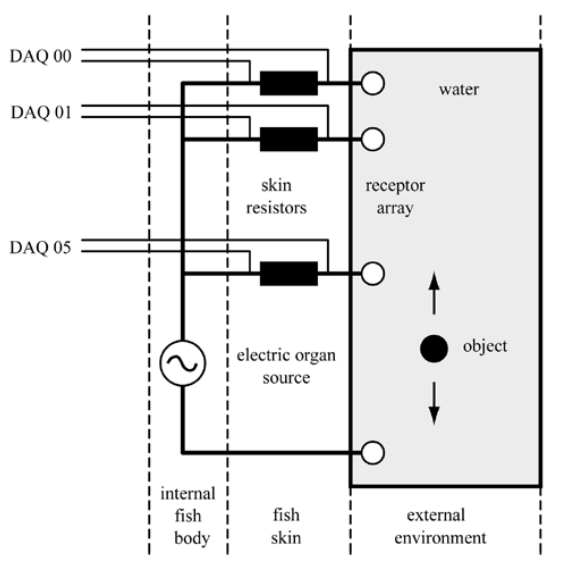

(b)

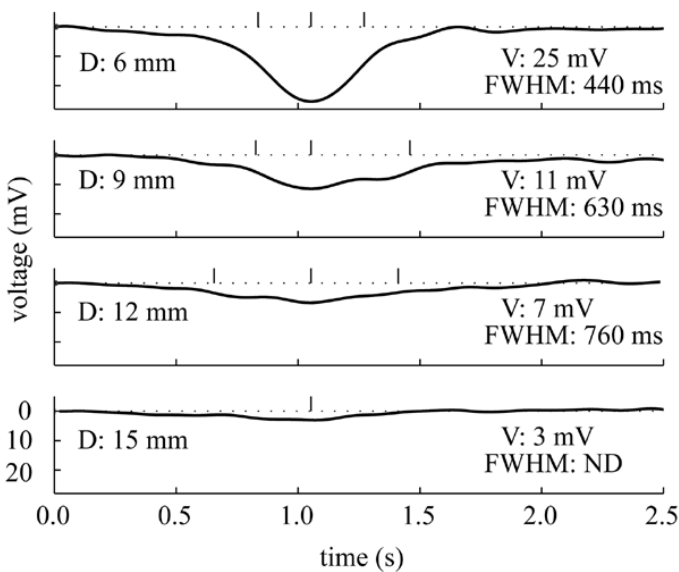

Fig. 5. Schematic of previously built electrosensor array and signals recorded from it, modified from [33]. (a) Schematic of the receptor array test setup. An electrolocation target was moved parallel to the submerged electrosensory array. Voltage signals, representing the transdermal potential experienced by each receptor, were recorded simultaneously from each channel as the target moved past the array. (b) Voltage changes recorded from an individual array element as an object is scanned past the array at four different distances. The object was a $1-\mathrm{cm}$-diameter plastic sphere. As the scan path was moved farther from the array, the peak amplitude of the perturbation decreased and the full-width at half-maximum (FWHM) increased. The FWHM for the furthest scan was not determined because of the low signal amplitude.

When fully developed, this sensing technology could provide a very responsive near-field sensor to guide autonomous navigation in cluttered environments. Most importantly, this sensor could require orders of magnitude less energy, and be more robust, than vision-based methods for obstacle detection and avoidance. For example, MBARI's Ventana ROV utilizes approximately $10^{3} \mathrm{~W}$ [3] to light its surroundings for visual sensing whereas a $10 \mathrm{~g}$ adult weakly electric fish $(13 \mathrm{~cm}$ long) utilizes approximately $10^{-2} \mathrm{~W}$ in total [35] some fraction of which is used to generate its electric field and transduce sensory signals. For a fair comparison, the sensing range of these two systems have to be taken into account, as well as the fact that the sensing field of the fish emanates in all directions, whereas the sensing field of the ROV is a small volume in front of the lights.

\section{RIBBON FIn PROPULSION FOR AUVS}

To better understand the fluid mechanical principles of knifefish ribbon fin propulsion, and to test its feasibility for further (a)

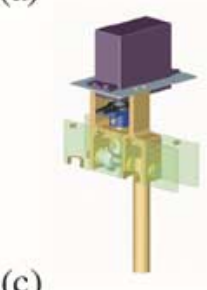

(b)

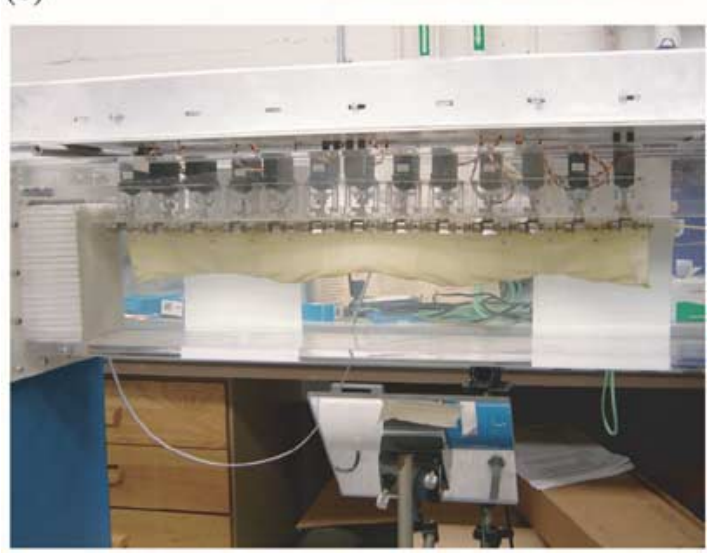

Fig. 6. (a) One of the 13 ribbon fin actuation elements. (b) Mounting structure for the array of actuation elements. (c) Robotic ribbon fin propelling itself in a flow tank. Length is $53 \mathrm{~cm}$. Movies of the ribbon fins in operation can be found at http://www.neuromech.northwestern.edu/publications.

development as an alternative propulsion method for underwater vehicles, a prototype of the fin was built (Fig. 6). Previous efforts in this area include the development of a $1 \mathrm{~m}$ long by $1 / 3 \mathrm{~m}$ deep undulating fin device with bending fin elements [36]. Here, our emphasis was to develop a ribbon fin structure as similar as possible to that used by black ghost knifefish, including the use of rigid fin elements. The amplitude of the traveling wave along the fin of knifefish is $\approx 5 \%$ of the total length of the fin [9], and the aspect ratio of the fin (chord/fin length) is $\approx 6-10 \%$. The value of the aspect ratio is difficult to measure exactly because there is no clear boundary on the actual fish where the rigid body ends and the undulating fin begins. Using the upper bound on the aspect ratio, the angular amplitude of the undulations is $\phi= \pm 30^{\circ}$. Although in some cases there are more than three full sinusoidal wavelengths along the fin, the typical number of wavelengths observed along the length of the fin is 2.5 [9]. We designed the mechanism for a maximum of three full wavelengths, with rays positioned every $90^{\circ}$ along the wavelength, resulting in a total of 13 actuator modules. Using commercially available hardware and servo motors to make our design as small as possible, the length of each module was determined to be $4.4 \mathrm{~cm}$. The total length of the fin was therefore $53 \mathrm{~cm}$, which dictated a fin depth of $d=0.05 \cdot 53 \cdot 6 / \pi=5 \mathrm{~cm}$.

In order to dynamically scale the fish's measured ribbbon fin undulation frequency of 1-3 Hz [9], a Reynolds number associated with the undulation was used. Assuming that the fin can be decomposed into $n$ independent flaps that represent the peak amplitudes of undulation, a chord length (i.e., width of each flap) can be calculated as $c=(l / n)$. Here, $l$ is the length of the fin and $n$ is twice the number of wavelengths present in the 
length of the fin. Using this as our characteristic length for the Reynolds number calculation

$$
\operatorname{Re}=\frac{2 c d \phi f}{\nu}
$$

where for a typical fish, $c=15 /(2 \cdot 2.5) \mathrm{cm}, d=1 \mathrm{~cm}, \phi=\pi / 3$ $\mathrm{rad}, f$ is the frequency of oscillation in hertz, and $\nu=0.01$ $\mathrm{cm}^{2} / \mathrm{s}$ is the kinematic viscosity of water. The frequency range of $1-3 \mathrm{~Hz}$ results in a Reynolds number range of 628-1885. For the robotic ribbon fin, designed for three full wavelengths, $c=53 /(2 \cdot 3) \mathrm{cm}$ and $d=5 \mathrm{~cm}$. Solving for $f$ gives a dynamically scaled frequency range of $0.06-0.2 \mathrm{~Hz}$. The digital servo motors used (HS-5645MG, Hitec RCD USA Inc., Poway, CA) are capable of applying $93 \mathrm{~N} \cdot \mathrm{cm}$ of torque at a frequency of 1.5 $\mathrm{Hz}$ and $\pm 30^{\circ}$ amplitude of oscillation, which was more than sufficient for driving the mechanism in the water. The motors were controlled using two servo controller cards (Mini SSC II Serial Servo Controller, Scott Edwards Electronics Inc., Sierra Vista, AZ) that convert serial commands from a PC into pulse-width modulated signals for the motors. Custom software written in MATLAB (The Mathworks, Natick, MA) was used to calculate the trajectory of each motor, and the data were continuously streamed to the controller cards through the serial port of the PC.

Initial results with this simple prototype have been encouraging. Using a simple sinusoidal traveling wave, it generated sufficient thrust to move itself through a flow tank. With further refinement it may provide a new propulsion technology for underwater vehicles in cases where precise movements at low speed are needed.

\section{Design IMPLICATIONS For AUVs}

Weakly electric fish, like most other animals, have evolved to fill a narrow ecological niche. Consequently, the mechanics and sensing abilities of the animal are exquisitely tuned to the behaviors it needs to survive. This specialization contrasts with the general purpose nature of most AUV designs. In order to obtain the power efficiency needed for long autonomous missions, it may be necessary to tune the vehicle design so that it can execute the most commonly needed maneuvers with a minimum of actuation and sensory power investment. For example, movements needed for oil rig inspection will be quite different from those needed for sea floor mapping.

From this basis, the movement capabilities and dynamics of the vehicle can be designed together with sensors that are needed to support the designed behaviors. For transient maneuvers such as those considered here, optimal control techniques and simple potential flow models may be applied to determine whether the behavioral goals correspond to efficient maneuvers of the vehicle, and if not, the vehicle design and sensor distribution can be carefully brought into correspondence with the behavioral objectives.

Given the high-power requirements of AUV sonar systems or ROV camera systems, consideration of the minimal sensing volume strategy utilized by weakly electric fish (sensing just enough space in the directions of movement to come to a stop) may also be useful. The approach is similar to one previously described for sensor-based motion planning in mobile robots [37]. Additional techniques observed in active sensing animals, such as the reduction of signal emission power as the range to the target of interest decreases (bats [38], dolphins [39]), may also be applied to reduce power consumption.

Finally, the unique sensing and locomotor mechanisms of the weakly electric fish, specially adapted for low speed maneuvering in complex and dark environments, may prove useful for application in future AUVs. Robotic ribbon fins may be placed along different parts of the AUV body to confer high precision translation and rotation. An artificial electrosensory array may be useful in murky water, cluttered environments, or operation in the dark. By generating a weak electric field around the AUV and placing sensors over the entire surface, an omnidirectional sensing volume would be obtained with many practical advantages for working in confined spaces. It would provide a sensory system with a mix of the features of smart skins and long range imaging sensors.

\section{CONCLUSION}

We have discussed the unique sensing and movement capabilities of the black ghost knifefish, emphasizing aspects of relevance to AUVs of the future. The black ghost knifefish, like the boxfish [40], provides an excellent example of the low-speed, high-maneuverability regime where biology has a competitive edge over current technology. We have taken some initial steps in analyzing this system, and we have discussed potential advantages of utilizing electrosense and ribbon fin propulsion in future AUVs.

\section{ACKNOWLEDGMENT}

The authors thank J. Radford for many useful discussions and help through a number of technical difficulties. They thank R. Murray and M. Milam for making NTG available for the optimal control analysis, and B. Dunbar for help with parameterizing the equations of motion and NTG coding. They thank E. Anderson for his fabrication and assembly of the ribbon fin prototype. They also thank M. Gharib for use of his facilities in testing the robotic ribbon fin.

\section{REFERENCES}

[1] D. Yoerger, J. Newman, and J.-J. Slotine, "Supervisory control system for the JASON ROV," IEEE J. Oceanic Eng., vol. OE-11, no. 3, pp. $392-400,1986$.

[2] H. Wang, R. Marks, T. McLain, S. Fleischer, D. Miles, G. Sapilewski, and S. Rock, "OTTER: A testbed submersible for robotics research," in ANS 1995, Monterey, CA, 1995.

[3] J. Rife and S. M. Rock, "A low energy sensor for AUV-based jellyfish tracking," in 12th Int. Symp. Unmanned Untethered Submersible Technology. Durham, NH, Aug. 2001.

[4] C. E. Carr, L. Maler, and E. Sas, "Peripheral organization and central projections of the electrosensory nerves in gymnotiform fish," J. Comp. Neurol., vol. 211, no. 2, pp. 139-153, 1982.

[5] M. A. MacIver, N. M. Sharabash, and M. E. Nelson, "Prey-capture behavior in gymnotid electric fish: Motion analysis and effects of water conductivity," J. Exp. Biol., vol. 204, no. 3, pp. 543-557, 2001.

[6] M. J. Lannoo and S. J. Lannoo, "Why do electric fishes swim backward? An hypothesis based on gymnotiform foraging behavior interpreted through sensory constraints," Env. Biol. Fishes, vol. 36, no. 2, pp. $157-165,1993$

[7] P. Nanjappa, L. Brand, and M. J. Lannoo, "Swimming patterns associated with foraging in phylogenetically and ecologically diverse American weakly electric teleosts (gymnotiformes)," Env. Biol. Fishes, vol. 58, no. 1 , pp. $97-104,2000$. 
[8] M. E. Nelson and M. A. MacIver, "Prey capture in the weakly electric fish Apteronotus albifrons: Sensory acquisition strategies and electrosensory consequences," J. Exp. Biol., vol. 202, no. 10, pp. 1195-1203, 1999.

[9] R. W. Blake, "Swimming in the electric eels and knifefishes," Can. J. Zool., vol. 61, pp. 1432-1441, 1983.

[10] "Electroreception and electrocommunication," J. Exp. Biol., vol. 202, no. 10,1999

[11] M. Sfakiotakis, D. M. Lane, and J. B. C. Davies, "Review of fish swimming modes for aquatic locomotion," IEEE J. Oceanic Eng., vol. 24, no. 2, pp. 237-252, 1999.

[12] M. A. MacIver, "The computational neuroethology of weakly electric fish: Body modeling, motion analysis, and sensory estimation," Ph.D. dissertation, Univ. of Illinois at Urbana-Champaign, May 2001.

[13] M. A. MacIver and M. E. Nelson, "Body modeling and model-based tracking for neuroethology," J. Neurosci. Meth., vol. 95, pp. 133-143, 2000

[14] M. W. Westneat, D. H. Thorsen, J. A. Walker, and M. E. Hale, "Structure, function and neural control of pectoral fins in fishes," IEEE J. Oceanic Eng., vol. 29, pp. 674-683, July 2004.

[15] M. M. Ellis, "The gymnotid eels of tropical America," Mem. Carnegie Museum, vol. 6, no. 3, pp. 109-195, 1913.

[16] J. G. Lundberg and F. Mago-Leccia, "A review of rhabdolichops (gymnotiformes, sternopygidae), a genus of South-American freshwater fishes, with descriptions of four new species," in Proc. Acad. Natural Sci. Phila., vol. 138, 1986, pp. 53-85.

[17] J. Bastian, "Pyramidal-cell plasticity in weakly electric fish: A mechanism for attenuating responses to reafferent electrosensory inputs," $J$. Comp. Physiol. A, vol. 176, no. 1, pp. 63-78, 1995.

[18] H. Lamb, Hydrodynamics, 6th ed. New York: Dover, 1932

[19] P. Holmes, J. Jenkins, and N. E. Leonard, "Dynamics of the Kirchhoff equations I: Coincident centers of gravity and buoyancy," Phys. D, vol. 118 , no. 3-4, pp. 311-342, 1998

[20] M. B. Milam, K. Mushambi, and R. M. Murray, "A new computational approach to real-time trajectory generation for constrained mechanical system," in Conf. Decision Control, 2000.

[21] M. B. Milam, "Real-time optimal trajectory generation for constrained dynamical systems," Ph.D. dissertation, California Inst. of Technology, 2003.

[22] G. L. Grabowsky, "Symmetry, locomotion, and the evolution of an anterior end-A lesson from sea-urchins," Evolution, vol. 48, no. 4, pp. 1130-1146, 1994

[23] R. G. Northcutt, "Understanding vertebrate brain evolution," Integr. Comp. Biol., vol. 42, no. 4, pp. 743-756, 2002.

[24] T. J. Koob and J. H. Long, "The vertebrate body axis: Evolution and mechanical function," Amer. Zool., vol. 40, no. 1, pp. 1-18, 2000.

[25] R. A. Dewel, "Colonial origin for eumetazoa: Major morphological transitions and the origin of bilaterian complexity," J. Morphol., vol. 243, no. 1 , pp. $35-74,2000$

[26] R. A. Hinde, Orientation, 2nd ed, ser. Animal behavior: A synthesis of ethology and comparative psychology. New York: McGraw-Hill, 1970 , ch. 7, pp. 146-192.

[27] D. Weihs, "Stability versus maneuverability in aquatic locomotion," Integr. Comp. Biol., vol. 42, no. 1, pp. 127-134, 2002

[28] J. J. Gibson, The Ecological Approach to Visual Perception. Boston, MA: Houghton-Mifflin, 1979.

[29] R. J. Full and D. E. Koditschek, "Templates and anchors: Neuromechanical hypotheses of legged locomotion on land," J. Exp. Biol., vol. 202, no. 23, pp. 3325-3332, 1999.

[30] H. J. Chiel and R. D. Beer, "The brain has a body: Adaptive behavior emerges from interactions of nervous system, body, and environment," Trends Neurosci., vol. 20, pp. 553-557, 1997.

[31] M. H. Dickinson, C. T. Farley, R. J. Full, M. A. R. Koehl, R. Kram, and S. Lehman, "How animals move: An integrative view," Science, vol. 288, no. 5463 , pp. $100-106,2000$

[32] Ö. Ekeberg, A. Lansner, and S. Grillner, "The neural control of fish swimming studied through numerical simulations," Adapt. Behav., vol. 3, no. 4, pp. 363-384, 1995.

[33] M. A. MacIver and M. E. Nelson, "Toward a biorobotic electrosensory system," Auton. Robots, vol. 11, no. 3, pp. 263-266, 2001.

[34] B. Rasnow, "The effects of simple objects on the electric field of Apteronotus," J. Comp. Physiol. A, vol. 178, no. 3, pp. 397-411, 1996.

[35] C. D. Hopkins, "Design features for electric communication," J. Exp. Biol., vol. 202, no. 10, pp. 1217-1228, 1999.
[36] M. Sfakiotakis, D. M. Lane, and B. C. Davies, "An experimental undulating-fin device using the parallel bellows actuator," in IEEE Int. Conf. Robotics Automation, Seoul, Korea, 2001.

[37] A. M. Shkel and V. J. Lumelsky, "Incorporating body dynamics into sensor-based motion planning: The maximum turn," IEEE Trans. Robot. Autom., vol. 13, no. 6, pp. 873-880, 1997.

[38] A. Boonman and G. Jones, "Intensity control during target approach in echolocating bats; stereotypical sensori-motor behavior in daubenton's bats, Myotis daubentonii," J. Exp. Biol., vol. 205, no. 18, pp. 2865-2874, 2002.

[39] W. W. L. Au and K. J. Benoit-Bird, "Automatic gain control in the echolocation system of dolphins," Nature, vol. 423, no. 6942, pp. 861-863, 2003.

[40] M. S. Gordon, J. R. Hove, P. W. Webb, and D. Weihs, "Boxfishes as unusually well-controlled autonomous underwater vehicles," Physiol. Biochem. Zool., vol. 73, no. 6, pp. 663-671, 2000.

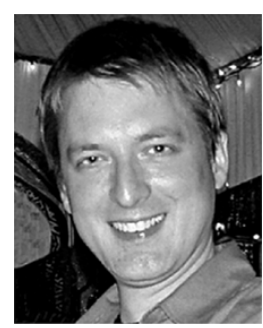

Malcolm A. MacIver received the B.Sc. degree in computer science and the M.A. degree in philosophy from the University of Toronto, Toronto, ON, Canada, in 1991 and 1992, respectively, and the $\mathrm{Ph} . \mathrm{D}$. degree in neuroscience from the University of Illinois at Urbana-Champaign in 2001.

He was a Postdoctoral Fellow in mechanical engineering and the Computation and Neural Systems Program at the California Institute of Technology, Pasadena, from 2001 to 2003. He joined Northwestern University, Evanston, IL, in 2003 with a joint appointment in the Department of Mechanical Engineering and the Department of Biomedical Engineering, as part of their Neural Engineering Initiative. His research interests are in the mechanical and neural basis of animal behavior, utilizing a variety of approaches spanning neuroethology, computer simulations, neurophysiology, biomechanics, and robotics.

Prof. MacIver is a member of the Society of Neuroscience and the International Society of Neuroethology.

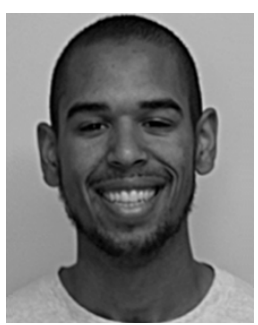

Ebraheem Fontaine received the B.S. degree from the Massachusetts Institute of Technology, Cambridge, in 2002 and the M.S. degree from the California Institute of Technology, Pasadena, in 2004, both in mechanical engineering. He is currently pursuing the Ph.D. degree in mechanical engineering at the California Institute of Technology.

His research interests include the kinematics and dynamics of biological creatures to inspire the design of novel robotics devices.

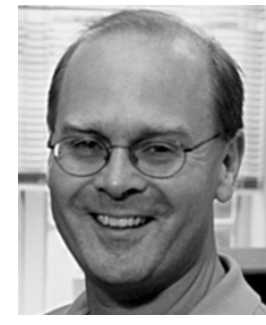

Joel W. Burdick received the undergraduate degrees in mechanical engineering and chemistry from Duke University, Durham, NC, in 1981 and the M.S. and $\mathrm{Ph} . \mathrm{D}$. degrees in mechanical engineering from Stanford University, Stanford, CA, in 1988

$\mathrm{He}$ joined the Department of Mechanical Engineering, California Institute of Technology, Pasadena, in 1988. He became Associate Professor in 1994 and Professor in 2000. He became a Professor of bioengineering in 2001. His current research interests include robotic locomotion, sensor-based robot motion planning, multifingered robotic hand manipulation, neural prosthetics, and applied nonlinear control theory.

Prof. Burdick received the National Science Foundation Presidential Young Investigator award, the Office of Naval Research Young Investigator award, and the Feynman fellowship. He was Program Chairman of IEEE/IROS 2001 and WAFR 2002. 